\title{
The Pharmacokinetics of Once-Daily Dosing With Gentamicin in Women With Postpartum Endometritis
}

\author{
J.A. Sunyecz, ${ }^{1}$ H.C. Wiesenfeld, ${ }^{2 *}$ and R.P. Heine ${ }^{2}$ \\ ${ }^{1}$ Uniontown, $P A$ \\ ${ }^{2}$ Department of Obstetrics, Gynecology, and Reproductive Sciences, University of Pittsburgh School of \\ Medicine/Magee-Womens Research Institute, Pittsburgh, PA
}

\begin{abstract}
Objective: To evaluate the pharmacokinetics and cost of once-daily dosing with gentamicin in women with postpartum endometritis.

Methods: Gentamicin in a single daily dose of $4.5 \mathrm{mg} / \mathrm{kg}$ was administered intravenously to 10 women with postpartum endometritis. Peak and trough gentamicin levels were measured, and nephrotoxicity and clinical ototoxicity were monitored. Pharmacokinetic data were analyzed, and a cost analysis of once-daily gentamicin administration was performed.

Results: The mean elimination constant was $0.105 \pm 0.008 \mathrm{~L} / \mathrm{h}$, and the mean volume of distribution was $0.34 \pm 0.07 \mathrm{~L} / \mathrm{kg}$. Mean peak gentamicin levels exceeded $11 \mathrm{mg} / \mathrm{L}$, and all trough levels were $<0.3 \mathrm{mg} / \mathrm{L}$. Cost savings of $44 \%$ were achieved with once-daily dosing of gentamicin, compared with traditional thrice-daily dosing.

Conclusions: Once-daily dosing with gentamicin in women with postpartum endometritis achieves therapeutic peak levels without drug accumulation. Substantial cost savings are realized with this dosing regimen. Infect. Dis. Obstet. Gynecol. 6:160-162, 1998. @ 1998 Wiley-Liss, Inc.
\end{abstract}

gentamicin; postpartum endometritis

G entamicin remains a popular bacteriocidal antibiotic for the treatment of infections caused by gram-negative organisms. Postpartum endometritis, typically a polymicrobial infection of both aerobic and anaerobic organisms, is often treated with clindamycin and gentamicin for broadspectrum coverage. While it is a highly effective antibiotic, gentamicin is associated with potentially serious toxicity, including nephrotoxicity and ototoxicity. A review of prospective clinical trials of adult patients treated with aminoglycosides between 1975 and 1982 indicated that the average frequencies of nephrotoxicity and ototoxicity of gentamicin are $14 \%$ and $8 \%$, respectively. ${ }^{1}$ Moreover, wide variations in dosage requirements of gentamicin have been demonstrated in the postpartum period, and subtherapeutic levels are seen in one third of women receiving standard dosing regimens. ${ }^{2,3}$

Aminoglycosides are commonly administered in either two or three divided doses per day. Two properties of aminoglycoside agents, the postantibiotic effect (PAE) and concentration-dependent killing, have raised interest in the idea of less frequent dosing of aminoglycosides. Postantibiotic effect is defined as ongoing bacterial inhibition after the antibiotic exposure has ceased. As such, the efficacy of aminoglycosides may not rely on maintaining serum concentrations above the minimum inhibitory concentration. ${ }^{4}$ The duration of the PAE

*Correspondence to: Dr. Harold C. Wiesenfeld, Dept. of Obstetrics, Gynecology, and Reproductive Sciences, MageeWomens Hospital, 300 Halket Street, Pittsburgh, PA 15213. E-mail: hwiesenfeld@mail.magee.edu 
correlates with the aminoglycoside concentration. Aminoglycoside antibiotics exhibit concentrationdependent bactericidal activity, with greater antibacterial activity seen in higher doses of the drug. ${ }^{4,5}$ In addition, a once-daily dosing regimen of aminoglycoside antibiotics may result in less drug accumulation in the renal cortex, thereby potentially decreasing nephrotoxicity. We report on the pharmacokinetics and cost of once-daily dosing with gentamicin in postpartum women with endometritis.

\section{SUBJECTS AND METHODS}

Women 18 years of age and older with postpartum endometritis were eligible for the study and were enrolled between July 1995 and January 1996. This study was approved by the institutional review board. The diagnosis of endometritis was based on temperature elevation greater than $38^{\circ} \mathrm{C}$ and uterine tenderness occurring at least 24 hours postpartum in the absence of any other source of infection. Exclusion criteria included aminoglycoside allergy, renal impairment (defined as serum creatinine $>1.2$ $\mathrm{mg} / \mathrm{dL}$ or estimated creatinine clearance $<60 \mathrm{~mL} /$ min), known hearing loss, or immunocompromised status. After obtaining informed consent, patients were administered a single dose of gentamicin at a dose of $4.5 \mathrm{mg}$ per kilogram of actual body weight over 30 minutes every 24 hours. Gentamicin was used in combination with clindamycin to provide polymicrobial coverage. Blood urea nitrogen and creatinine were determined prior to initiation of therapy and every 48 hours during treatment. Nephrotoxicity was defined as an elevation of serum creatinine from baseline greater than $0.5 \mathrm{mg} /$ dL. Gentamicin levels were drawn 30 minutes after completion of the first and second doses, $30 \mathrm{~min}$ utes prior to the second and third doses, and an 8-hour level was drawn after the second dose. Pharmacokinetic data were analyzed for each patient using standard pharmacokinetic principles. ${ }^{6}$ Ototoxicity was evaluated clinically by regularly questioning patients about hearing loss, tinnitus, and vertigo. No audiometric evaluations were performed.

Cost analysis was based on 72 hours of treatment using $300 \mathrm{mg} / \mathrm{d}$ of gentamicin in the once-daily regimen compared with $100 \mathrm{mg}$ every 8 hours in the traditional dosing regimen. Patient charges from the pharmacy for preparation and dispensing
TABLE I. Demographic and pharmacokinetic data

\begin{tabular}{lcc}
\hline Characteristic & Mean $\pm \mathrm{SD}^{\mathrm{a}}$ & Range \\
\hline Age $(\mathrm{y})$ & $26.2 \pm 8.4$ & $18-4 \mid$ \\
Weight $(\mathrm{kg})$ & $80.3 \pm 23.9$ & $56-136$ \\
Dose $(\mathrm{mg})$ & $360 \pm 105$ & $255-600$ \\
Half life $(\mathrm{h})$ & $4.36 \pm 0.22$ & $3.96-4.67$ \\
Estimated clearance $(\mathrm{mL} / \mathrm{min})$ & $149 \pm 53$ & $84-271$ \\
\hline
\end{tabular}

aSD, standard deviation.

and nursing administration times were calculated. Nursing costs were based on hospital costs and were determined using an average registered nurse wage of $\$ 18 / \mathrm{h}$. Charges for intravenous tubing and gentamicin level monitoring were excluded in the cost analysis, as intravenous tubing costs are minimal, and gentamicin level monitoring may be necessary with both single and thrice-daily treatment regimens.

Gentamicin serum concentrations were determined by fluorescence polarization immunoassay using an automated fluorescence polarization analyzer. (Abbott Diagnostics, Abbott Park, IL). For levels between 2 and $5 \mathrm{mg} / \mathrm{L}$, the coefficient of variation was $5 \%$. Levels between 10 and $15 \mathrm{mg} / \mathrm{L}$ had a coefficient of variation of $6 \%$.

\section{RESULTS}

Ten women with postpartum endometritis were enrolled in this study. Table 1 summarizes demographic data as well as half life and estimated creatinine clearance. The mean $( \pm$ standard deviation [SD]) elimination constant was $0.105 \pm 0.008 \mathrm{~L} / \mathrm{h}$, with a range of $0.096-0.122 \mathrm{~L} / \mathrm{h}$. The mean volume of distribution $( \pm \mathrm{SD}$ ) was $0.34 \pm 0.07 \mathrm{~L} / \mathrm{kg}$, with a range of $0.27-0.51 \mathrm{~L} / \mathrm{kg}$. The mean first peak level $( \pm \mathrm{SD})$ was $11.6 \pm 2.3 \mathrm{mg} / \mathrm{L}$. The mean second peak level $( \pm \mathrm{SD})$ was $13.0 \pm 2.5 \mathrm{mg} / \mathrm{L}$. All first and second trough levels were less than $0.3 \mathrm{mg} / \mathrm{L}$. A favorable clinical response was seen in nine patients. The median duration of therapy was 3 days. One patient was administered heparin therapy for presumed septic pelvic thrombophlebitis. There was no nephrotoxicity nor clinical ototoxicity detected.

Based on a 3-day course of gentamicin, the patient charge from our pharmacy for the once-daily regimen was $\$ 241$, compared with $\$ 422$ for the thrice-daily regimen. After adding nursing administration times, the total patient charges were $\$ 250$ and $\$ 449$ respectively. This represents a $44 \%$ re- 
duction in patient charges for the once-daily regimen when compared with the thrice-daily regimen for 72 hours of treatment.

\section{DISCUSSION}

This study demonstrates several important points regarding a once-daily dose of gentamicin among postpartum women. Our data show that no drug accumulation occurred during our treatment period, as trough levels were consistently less than 0.3 $\mathrm{mg} / \mathrm{L}$. This is important clinically as data suggests that aminoglycoside-free periods during therapy are necessary to reduce the risk of nephrotoxicity and ototoxicity. ${ }^{7}$ We were also able to consistently achieve elevated peak levels with our dosing regimen, optimizing the PAE and concentrationdependent killing characteristics of aminoglycoside antibiotics. ${ }^{7}$ Our pharmacokinetic parameters were consistent with published data from Sangha et al. evaluating once-daily dosing with gentamicin in intensive care unit patients with open fractures. ${ }^{8}$ The numerous physiologic changes that occur during the postpartum period may cause wide fluctuations in the pharmacokinetic parameters. This may explain the differences in elimination rate constant, volume of distribution, and half life between our data and those reported recently by Del Priore et al. ${ }^{9}$ An additional factor that may reflect a higher half life is that our laboratory could not detect gentamicin levels less than $0.3 \mathrm{mg} / \mathrm{L}$, the trough value used to calculate the half life. Higher trough levels lengthen the half life. Finally, we were able to demonstrate significant cost savings with a oncedaily regimen in the postpartum patient with endometritis, reducing charges by nearly one half. The results of this study support the conclusions of two reports of once-daily gentamicin in the treat- ment of puerperal uterine infections. ${ }^{9,10}$ Oncedaily dosing of gentamicin for the treatment of postpartum endometritis is efficacious and costeffective, and it appears that this regimen is not associated with a high incidence of nephrotoxicity or ototoxicity.

\section{REFERENCES}

1. Kahlmeter G, Dahlager JL: Aminoglycoside toxicity: a review of clinical studies published between 1975 and 1982. J Antimicrob Chemother 13(suppl A):9-22, 1984.

2. Zaske DE, Cipollo RJ, Strate RG, et al.: Rapid gentamicin elimination in obstetric patients. Obstet Gynecol 56:559-563, 1980.

3. Duff P, Jorgensen JH, Gibbs RS, Blanco JD, Alexander $G$, Castaneda YS: Serum gentamicin levels in patients with post cesarean endomyometritis. Obstet Gynecol 61:723-727, 1983.

4. Gilbert DN: Once daily aminoglycoside therapy. Antimicrob Agents Chemother 35:399-403, 1991.

5. Prins JM, Buller HR, Kuijper EJ, Tange RA, Speelman P: Once versus thrice daily gentamicin in patients with serious infections. Lancet 341:335-339, 1993.

6. Notari RE: Biopharmaceutics and Clinical Pharmacokinetics. 4th ed. New York: Marcel Dekker, Inc, 1987.

7. Dew RB, Susla GM: Once daily aminoglycoside treatment. Infect Dis Clin Pract 5:12-24, 1996.

8. Sangha KS, Miyagawa CI, Healy DP, Bjornsn HS: Pharmacokinetics of once-daily dosing of gentamicin in surgical intensive care unit patients with open fractures. Ann Pharmacother 29:117-119, 1995.

9. Del Priore G, Jackson-Stone M, Shim EK, Garfinkel J, Eichmann MA, Frederiksen MC: A comparison of once daily and 8-hour gentamicin dosing in the treatment of postpartum endometritis. Obstet Gynecol 87:994-1000, 1996.

10. Mitra AG, Whitten MK, Laurent SL, Anderson WE: A randomized, prospective study comparing once-daily gentamicin versus thrice-daily gentamicin in the treatment of puerperal infection. Am J Obstet Gynecol 177: 786-791, 1997. 


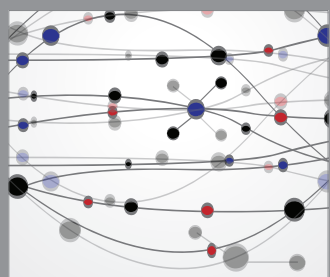

The Scientific World Journal
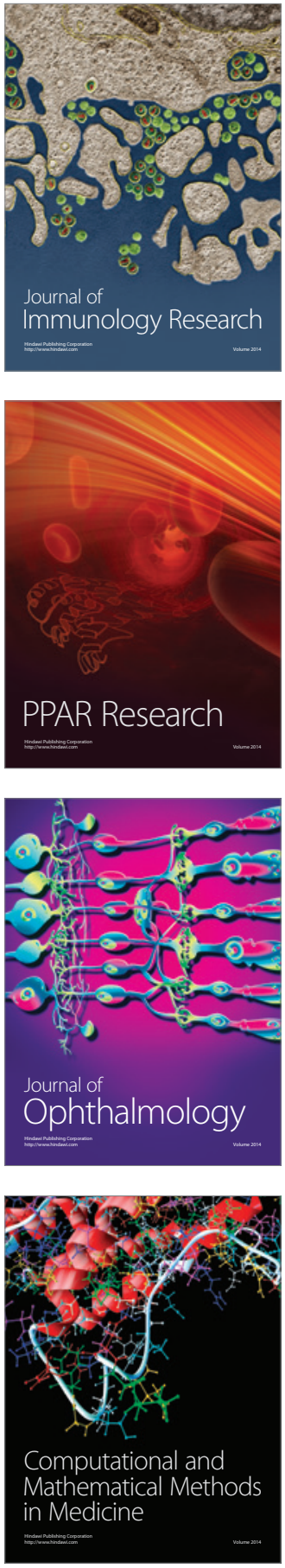

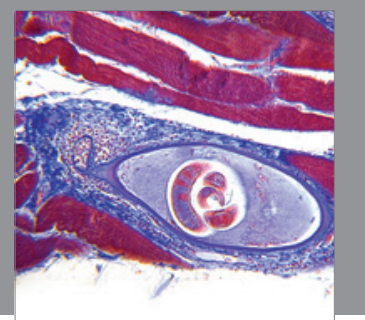

Gastroenterology

Research and Practice
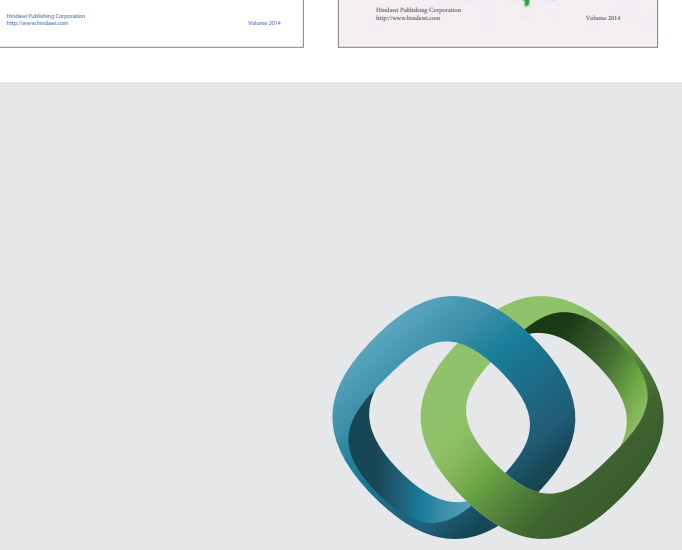

\section{Hindawi}

Submit your manuscripts at

http://www.hindawi.com
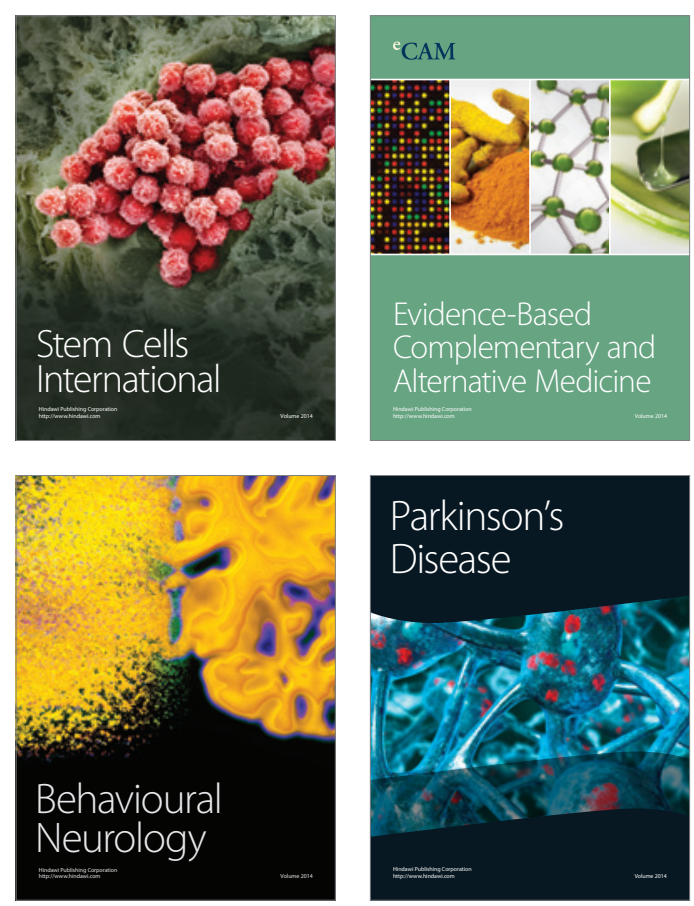

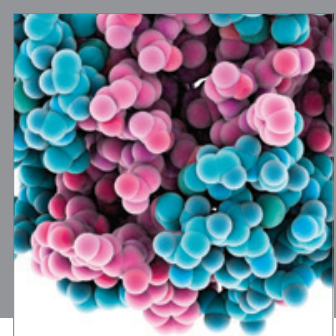

Journal of
Diabetes Research

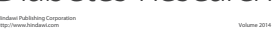

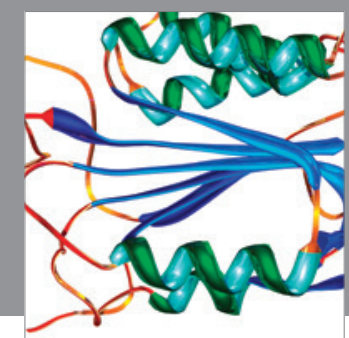

Disease Markers
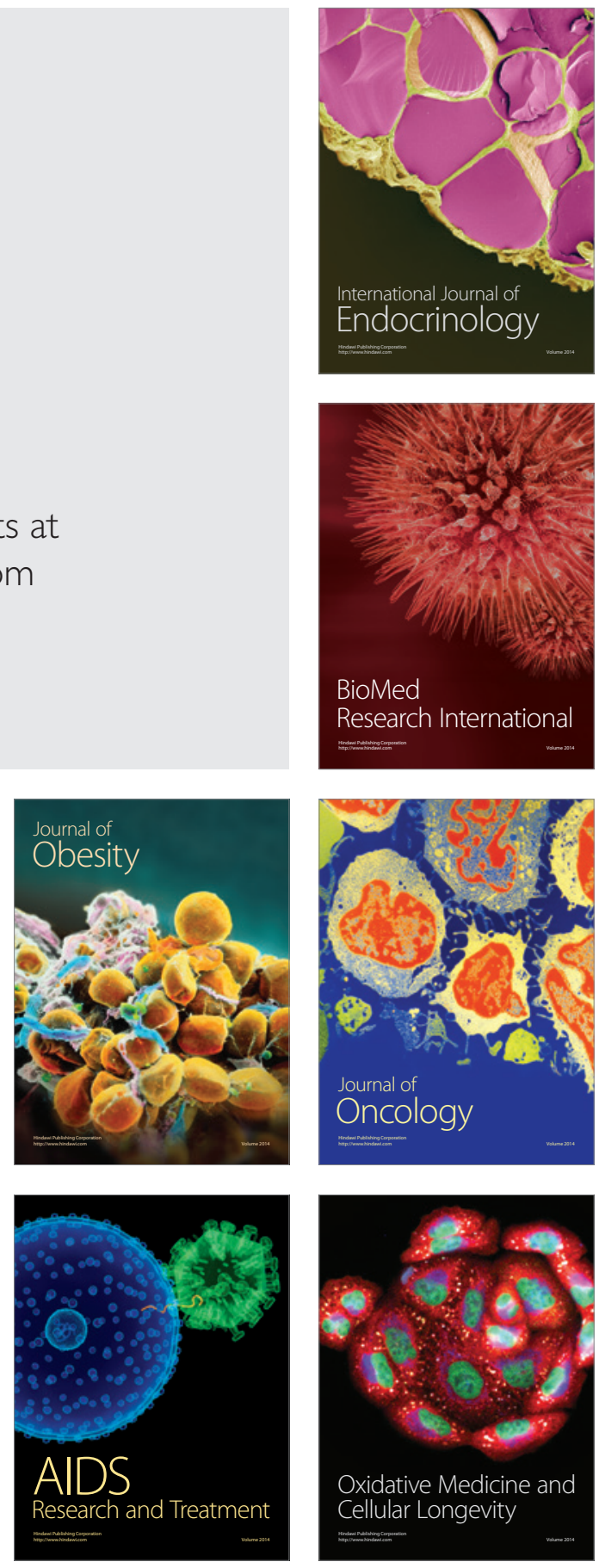